\title{
Optimization in Oil Pipelines Arrangement with Fermat Problem
}

\author{
Dong-Ping WEI ${ }^{1,2, a}$, Hong-Ya ZHAO ${ }^{1, b}$, Tian-Li LEI ${ }^{1, c,{ }^{*}}$ \\ ${ }^{1}$ Math and Physics Department, Shenzhen Polytechnic, Shenzhen, 518055, China \\ ${ }^{2}$ School of Management, Shanghai University, Shanghai, 200444, China \\ awdp@szpt.edu.cn, bhy.zhao@szpt.edu.cn, ${ }^{\mathrm{a}}$ It|@szpt.edu.cn \\ ${ }^{*}$ Corresponding author
}

Keywords: Fermat Problem, Optimization Gas or Oil Pipelines.

\begin{abstract}
The problem of optimization gas or oil pipelines arrangement can be converted into Fermat problem of "Two points and One Line". The optimal arrangement of pipelines with the same unit cost of shared or non - shared pipeline (OAPSU-Model) is proposed to study the problems of lowest cost of pipelines firstly. The optimal arrangement of pipelines with the different unit cost of shared or non shared pipeline (OAPDU-Model) is established to find the Fermat point and the minimum cost of pipelines. In fact, OAPSU-Model is the special case of OAPDU-Model.
\end{abstract}

\section{Introduction}

An oilfield company plans to build two refineries on the one side of railway, and built a station in the railway to transport oil products as shown in Fig.1. Some Oilfield Design Institutes hope to establish the general mathematical models and methods to cut pipelines construction costs as much as possible. These kinds of problem are common in oil and gas transportation. Actually, this kind of problem is very complicated because the distance between refineries and the vertical distance between refineries and railway are different in different locations. And some position is not suitable to build refineries or railway stations. And the unit cost of shared or non-shared pipelines is different because the materials or sizes of pipes are different. The problems are interesting for many scholars. For example, Wang Yuchun built a mathematical model based on the correlation method of gray system to study pipelines construction problem [1].And WU Ming set up a running model to long distance heated crude oil pipeline [2]. However, the gas and oil transportation problems require further study in different case. Hence, it is valuable to propose the mathematical model based on Fermat problem to study this kind of transportation problems.

\section{Notations}

$k:$ The unit cost of shared or non-shared pipelines

$f:$ The total length of pipelines

$F:$ The total cost of pipelines

$a$ : The vertical distance from refinery A to the railway

$b:$ The vertical distance from refinery $\mathrm{B}$ to the railway

$l$ : The horizontal distance between refinery A and $\mathrm{B}$

\section{Methodologies}

\section{OAPSU-Model}

The positions of A, B refineries and railway can be shown in Fig.1. AC is defined as $\mathrm{y}-$ axis. The railway is defined as $\mathrm{X}-$ axis. $\mathrm{C}$ is defined as origin. Hence, the coordinate system can be established as Fig.1. 
The coordinate of refinery $A$ is $(0, a)$. The coordinate of refinery $B$ is $(\boldsymbol{l}, \boldsymbol{b})$. And it is reasonable to assume $b \geq a$. $P A$ is the pipelines from $A$ to $P, P B$ is the pipelines from $B$ to $P$. And $P(x, y)$ is the common points of pipelines of $P A$ and $P B . E$ is the railway station. $P E$ is the shared pipelines for $A, B$ refineries. $A^{\prime}$ is the symmetry point of $A$ with the standard line $L_{1}$. Given two point A, B, and a line $\mathrm{X}$ - axis on one plane, the problem is to find a point $P$ where the total distance of $P A, P B, P E$ is minimal. Actually, this problem is the evolution of the historical mathematics famous Fermat problem [3]. $P$ is the Fermat point.

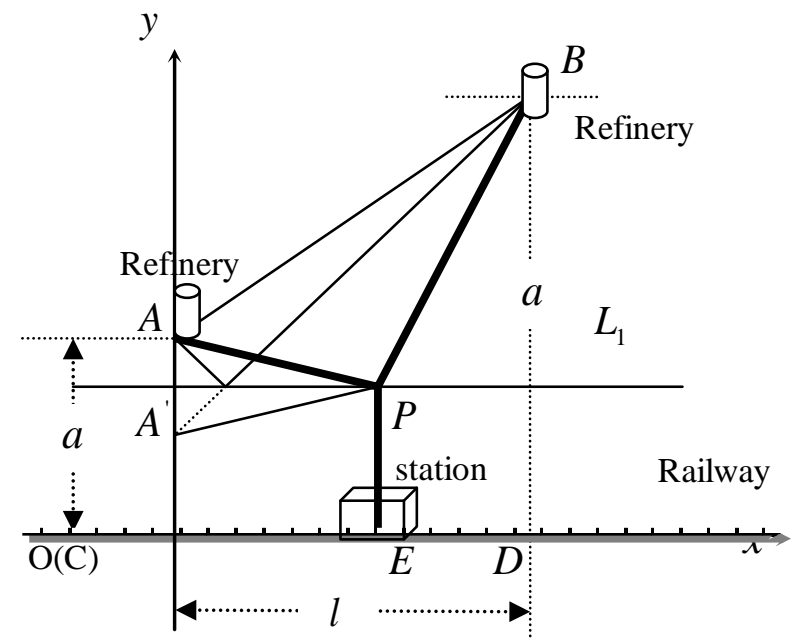

Fig. 1 The arrangement of pipelines

The unit cost of shared or non - shared pipelines is the same, then the total cost of pipelines is:

$F(x, y)=k \cdot f(x, y)$

where $k$ is the unit cost of shared and non-shared pipelines. $f$ the total length of pipelines can be obtained as following

$f=A P+B P+P E=A^{\prime} P+B P+P E \geq A^{\prime} B+P E=\sqrt{l^{2}+(2 y-a-b)^{2}}+y$

Where $y \in[0, a]$ the proof can be found in the document [4] .

The Optimal arrangement of pipelines with same unit cost of shared and non-shared pipelines ( OAPSU-Model) can be described as following

$\min F=k\left[\sqrt{l^{2}+(2 y-a-b)^{2}}+y\right]$

s.t. $\quad 0 \leq y \leq a$

According to the first order condition of maximization [5], it can obtain

$F^{\prime}=k\left[\frac{2(2 y-a-b)^{2}}{\sqrt{l^{2}+(2 y-a-b)^{2}}}+1\right]$

The optimal point can be obtained by $F^{\prime}=0$ as following 
$y=\frac{a+b}{2}-\frac{\sqrt{3} l}{6}$ or $y=\frac{a+b}{2}+\frac{\sqrt{3} l}{6}>a$

where the second solution $y=\frac{a+b}{2}+\frac{\sqrt{3} l}{6}>a$ should be abandoned because $0 \leq y \leq a$. And the following inequality can be obtained by the first solution

$0 \leq \frac{a+b}{2}-\frac{\sqrt{3} l}{6} \leq a \Rightarrow \sqrt{3}(b-a) \leq l \leq \sqrt{3}(a+b)$

Equation (6) indicates that the relations of the distance between $A, B$ and railway and the horizontal distance between $A, B$ influence on the Fermat point $P$ critically. The detailed relations can be discussed as following

1) If the horizontal distance $l$ of A, B refinery satisfies $0<l<\sqrt{3}(b-a)$, then $F^{\prime}(y)<0$ and $F(y)$ is decreasing in $[0, a]$ monotonically. Therefore, when $P$ and $A$ coincide with each other, the shortest line is obtained. The minimum cost of pipelines can be gained by following

$$
F_{\min }=\left[a+\sqrt{l^{2}+(a-b)^{2}}\right] k
$$

where the coordinate $P$ is $(0, a)$.

2) When the horizontal distance $l$ of $A, B$ refineries satisfies $\sqrt{3}(b-a) \leq l \leq \sqrt{3}(b+a)$, then $F(y)$ is decreasing in $\left[0, \frac{a+b}{2}-\frac{\sqrt{3} l}{6}\right]$ and increasing in $\left[\frac{a+b}{2}-\frac{\sqrt{3} l}{6}, a\right]$ monotonically. Therefore, when the Coordinates of $P$ is $\left(\frac{\sqrt{3}(a-b)+l}{2}, \frac{a+b}{2}-\frac{\sqrt{3} l}{6}\right)$, the minimum total cost of pipelines can be obtained by following

$$
F_{\min }=\frac{a+b+\sqrt{3} l}{2} \cdot k
$$

3)When the horizontal distance $l$ of $A, B$ refineries satisfies $l>\sqrt{3}(a+b)$, then $F(y)$ is monotonically increasing in $[0, a]$. Therefore, when the Coordinates of $P$ is $\left(\frac{a l}{a+b}, 0\right)$, the minimum total cost of pipelines can be obtained by following

$F_{\text {min }}=\sqrt{l^{2}+(a-b)^{2}} \cdot k$

\section{OAPDU-Model}

$k_{1}$ is the unit cost of non-shared pipelines. $k_{2}$ is the unit cost of shared pipelines. Thus the total cost of pipelines is

$$
F=k_{1}(P A+P B)+k_{2} P E=k_{1} \sqrt{l^{2}+(2 y-a-b)^{2}}+k_{2} y
$$

The optimal arrangement of pipelines with the different unit cost of shared or non - shared pipeline (OAPDU-Model) can be obtained as following 
$\min F=k_{1} \sqrt{l^{2}+(2 y-a-b)^{2}}+k_{2} y$

(11)

s.t. $\quad(0 \leq y \leq a)$

According to the first order condition of maximization [5], it can get

$F^{\prime}=\frac{2 k_{1}(2 y-a-b)}{\sqrt{l^{2}+(2 y-a-b)^{2}}}+k_{2}$

Setting $F^{\prime}=0$, it is easy to obtain $k_{2}^{2} l^{2}+\left(k_{2}^{2}-4 k_{1}^{2}\right)(2 y-a-b)^{2}=0$

$$
y_{1}=\frac{1}{2}(a+b)+\frac{k_{2} l}{2 \sqrt{4 k_{1}^{2}-k_{2}^{2}}}
$$

$$
y_{2}=\frac{1}{2}(a+b)-\frac{k_{2} l}{2 \sqrt{4 k_{1}^{2}-k_{2}^{2}}}
$$

If $k_{1}=k_{2}$, then $y_{1}=\frac{1}{2}(a+b)+\frac{\sqrt{3}}{6} l, y_{2}=\frac{1}{2}(a+b)-\frac{\sqrt{3}}{6} l$. In that case, the result of OAPSU-Model is consistent with OAPDU-Model. In another words, OAPSU-Model is the special case of OAPDU-Model.

When $k_{1} \neq k_{2}$, it can obtain $0<k_{1}<2 k_{2}$ due to $4 k_{1}{ }^{2}-k_{2}{ }^{2}>0$. It means that the cost of shared pipelines should not exceed 2 times that of non-shared pipelines. Otherwise, the two refineries should be arranged two pipelines to railway respectively.

Because $0 \leq y \leq a$, it is easy to obtain $0 \leq \frac{1}{2}(a+b)-\frac{k_{2} l}{2 \sqrt{4 k_{1}{ }^{2}-k_{2}^{2}}} \leq a$, the scope of $l$ can be obtained by following inequality

$$
\frac{(b-a) \sqrt{4 k_{1}^{2}-k_{2}^{2}}}{k_{2}} \leq l \leq \frac{(a+b) \sqrt{4 k_{1}^{2}-k_{2}^{2}}}{k_{2}}
$$

The following discussions show how $l$ effects on Ferma point $P$.

1)When $0 \leq l \leq \frac{(b-a) \sqrt{4 k_{1}{ }^{2}-k_{2}^{2}}}{k_{2}}, \quad F^{\prime}(y)<0 \quad . \quad F(y)$ is monotonically decreasing in $[0, a]$.Therefore,the lowest total cost of pipelines can be obtained by following equation:

$$
F_{\min }=k_{1} \sqrt{l^{2}+(a-b)^{2}}+k_{2} a
$$


where the coordinate of $P$ is $(0, a)$.

2)When $\frac{(b-a) \sqrt{4 k_{1}^{2}-k_{2}^{2}}}{k_{2}} \leq l \leq \frac{(a+b) \sqrt{4 k_{1}^{2}-k_{2}^{2}}}{k_{2}}$, the minimum cost of pipelines $F(y)$ achieves at the point $y=\frac{1}{2}(a+b)-\frac{k_{2} l}{2 \sqrt{4 k_{1}{ }^{2}-k_{2}^{2}}}$. Thus the coordinates of $P$ is $\left(\frac{(b-a) \sqrt{4 k_{1}^{2}-k_{2}^{2}}+l}{2}, \frac{1}{2}(a+b)-\frac{k_{2} l}{2 \sqrt{4 k_{1}^{2}-k_{2}^{2}}}\right)$. The lowest total cost of pipelines can be obtained by following equation:

$F_{\text {min }}=\frac{1}{2}(a+b) k_{2}+\frac{\sqrt{4 k_{1}^{2}-k_{2}^{2}}}{2} l$

3)When $l>\frac{\sqrt{4 k_{1}^{2}-k_{2}^{2}}}{k_{1}}(a+b)$,it's easy to see that $f(y)$ is increasing in $[0, a]$. Thus the Coordinates of $P$ is $\left(\frac{a l}{b+a}, 0\right)$. In that case, the lowest total cost of pipelines can be obtained by following equation:

$F_{\text {min }}=k_{1} \sqrt{l^{2}+(a+b)^{2}}$

It is easy to conclude that OAPDU-Model can be degraded into OAPSU-Model. For example, when $k_{1}=k_{2}$, Equation (17) can get following result

$F_{\min }=\frac{1}{2}(a+b) k_{1}+\frac{\sqrt{3} k_{1}}{2} l=\frac{a+b+\sqrt{3}}{2} l$

\section{Conclusions}

The optimal arrangement of pipelines with the different unit cost of shared or non - shared pipeline (OAPSU-Model) is proposed to study the problems of lowest cost of pipelines construction projects. The cost of whole pipelines $F_{\min }$ can be obtained by OAPDU-Model easily. And it is easy to get the optimal common point $P$. When the horizontal distance $l$ of the two oil refineries satisfies the three different conditions including $0<l<\sqrt{3}(a+b), \sqrt{3}(b-a)<l<\sqrt{3}(a+b), l>\sqrt{3}(a+b)$, the lowest cost of whole pipelines $F_{\min }$ and the optimal common point $P$ are shown in Table 1. 
Table $1 \quad F_{\min }$ and $P$ with OAPSU-Model

\begin{tabular}{lll}
\hline The relations of $l, a$ and $b$ & The coordinate of point $P$ & $F_{\text {min }}$ \\
\hline $0<l<\sqrt{3}(b-a)$ & $(0, a)$ & {$\left[a+\sqrt{l^{2}+(a-b)^{2}}\right] k$} \\
$\sqrt{3}(b-a) \leq l \leq \sqrt{3}(b+a)$ & $\left(\frac{\sqrt{3}(a-b)+l}{2}, \frac{a+b}{2}-\frac{\sqrt{3} l}{6}\right)$ & $\frac{a+b+\sqrt{3} l}{2} \cdot k$ \\
$l>\sqrt{3}(a+b)$ & $\left(\frac{a l}{a+b}, 0\right)$ & $\sqrt{l^{2}+(a-b)^{2}} \cdot k$
\end{tabular}

OAPDU-Model is proposed to the optimal arrangement of pipelines with the different unit cost of shared or non-shared pipeline. The cost of whole pipelines $F_{\text {min }}$ achieves at the point $y=\frac{1}{2}(a+b)-\frac{k_{2} l}{2 \sqrt{4 k_{1}^{2}+k_{2}^{2}}}$. It is easy to conclude that OAPDU-Model can simplify into OAPSU-Model when $k_{1}=k_{2}$. When $0<k_{2} \leq 2 k_{1}$, the horizontal distance of two refineries and the relations of $a$ and $b$ satisfy the following conditions, the lowest cost of whole pipelines $F_{\min }$ and the optimal common point $P$ are shown in Table 2.

Table $2 \quad F_{\min }$ and $P$ with OAPDU-Model

\begin{tabular}{lll}
\hline The relations of $l, a$ and $b$ & The coordinate of point $P$ & $F_{\min }$ \\
\hline $0 \leq l \leq \frac{(b-a) \sqrt{4 k_{1}^{2}-k_{2}^{2}}}{k_{2}}$ & $(0, a)$ & $k_{1} \sqrt{l^{2}+(a-b)^{2}}+k_{2} a$ \\
$\frac{(b-a) \sqrt{4 k_{1}{ }^{2}-k_{2}{ }^{2}}}{k_{2}} \leq l \leq \frac{(a+b) \sqrt{4 k_{1}{ }^{2}-k_{2}{ }^{2}}}{k_{2}}$ & $\left(\frac{(b-a) \sqrt{4 k_{1}{ }^{2}-k_{2}{ }^{2}}+l}{2}, \frac{1}{2}(a+b)-\frac{k_{2} l}{2 \sqrt{4 k_{1}{ }^{2}-k_{2}{ }^{2}}}\right)$ & $\frac{1}{2}(a+b) k_{2}+\frac{\sqrt{4 k_{1}^{2}-k_{2}^{2}}}{2} l$ \\
$l>\frac{\sqrt{4 k_{1}^{2}-k_{2}^{2}}}{k_{1}}(a+b)$ & $\left(\frac{a l}{b+a}, 0\right)$ & $k_{1} \sqrt{l^{2}+(a+b)^{2}}$ \\
\hline
\end{tabular}

\section{Acknowledgements}

This work is supported by National Natural Science Funds of China (Grant No. 31100958) and Youth Innovation fund of Shenzhen polytechnic (Grant No. 601422K35018) and the Project of Higher Vocational Education Research Society of China (Grant No.GZYLX1213345) and Shenzhen Education Society (Grant No.XH021). And the authors would like to thank the editor and an anonymous referee for their helpful comments on the manuscript.

\section{References}

[1] Yuchun W, Optimization in Oil and Gas Pipeline with Gray System Method, Petroleum Planning \& Engineering, 1993, 3: 10-12.

[2] WU M, WANG J, LIU J, et al., Optimization of Running Scheme With Long Distance Heated Crude Oil Pipeline, Journal of Liaoning University of Petroleum \& Chemical Technology, 2006, 1: 017.

[3] Kuhn H W, A note on Fermat's problem, Mathematical programming, 1973, 4(1): 98-107.

[4]Shu B,N, A Generalization of Fermat point in triangle, High School Mathematical Teaching ,2006,5:32-33.

[5]Widder D V, Advanced calculus, Courier Dover Publications, 2012. 